\title{
Microstructure and electrical transport in electrodeposited Bi films
}

\author{
J. Moral-Vico ${ }^{\mathrm{a}, *}$, N. Casañ-Pastor ${ }^{\mathrm{a},}{ }^{\dagger}$, A. Camón ${ }^{\mathrm{b}}$, C. Pobes ${ }^{\mathrm{b}}$, R.M. Jáudenes ${ }^{\mathrm{b}}$, P. Strichovanec ${ }^{\mathrm{b}}$ \\ and L. Fàbrega ${ }^{a}, \dagger$
}

a ICMAB-CSIC, Campus de la UAB, E-08193 Bellaterra, Barcelona, Spain.

${ }^{\mathrm{b}}$ ICMA-CSIC-Universidad de Zaragoza, Pedro Cerbuna 12, E-50009 Zaragoza, Spain.

*Corresponding author, e-mail address: antoniojavier.moral@uab.cat

$\dagger$ Equal contribution

\begin{abstract}
The semimetal character of bismuth and its large photon absorbing power make of this element the most suitable absorber material for X-ray low temperature detectors. This application requires coatings of $\mathrm{Bi}$ with thicknesses and properties that only electrodeposition methods may achieve. Although there are studies on electrodeposition of bismuth for these detectors and other devices, the process is not straightforward and has not been sufficiently studied in terms of the desired final properties, neither the effect of different parameters is well known or easily reproduced. This work reports the influence of two different electrolytes, of the deposition method, and of heating and stirring on the structure, microstructure and transport properties of bismuth films. Typically, rhombohedral $\mathrm{Bi}$ is obtained upon electrodeposition with very good crystallinity, and some crystal preferential orientation, while significant empirical correlations are found among electrochemical parameters, microstructure, and resistivity. Such correlation allows the identification of the deposition parameters for coatings that yield the optimal functional properties.
\end{abstract}

KEYWORDS: Bismuth, electrodeposition, resistivity, low temperature detectors. 


\section{Introduction}

Bismuth is an element with most unusual electronic properties. It is a semimetal, with highly anisotropic Fermi surface, low carrier densities and very large carrier mobilities; it also displays large positive magnetoresistance [1], and bulk superconductivity has been reported very recently [2]. Due to these peculiarities, Bi has motivated much interest in condensed matter research and also a significant number of applications, especially in the form of films. It has been used to test several quantum confinement phenomena [3-7], and among the applications of Bi films are magnetic field and current sensing [1,5,8], electrochromic and fotovoltaic devices [9-12], thermoelectric devices [13], and electrodes for a variety of appliances [14-16].

Because of its large atomic number, semimetallic character, and very low heat capacity, bismuth is also a most suitable material for X-ray absorbers in extremely sensitive radiation detectors, such as microcalorimeters based on Transition Edge Sensors (TES) [17-22]. To achieve high quantum efficiency (that is, high photon absorption) up to energies of $10 \mathrm{keV}$, Bi coatings are required to be several microns thick [17-19]. Also, films require being compact and uniform. On the other hand, it is known that Bi films display usually a semiconductor-like behaviour [3,19,20,22-25], instead of the typical $\rho(\mathrm{T})$ of semimetallic bulk bismuth; post deposition annealing has been shown to improve morphology and resistivity $[5,19]$ but such thermal treatments cannot be used with TES devices, since they would damage them. Thus, and since these detectors are meant to operate at extremely low temperatures, a high thermal conductivity at the operation temperature requires a $\rho(T)$ as close as possible to bulk, which could be achieved through a careful control of defects and microstructure of the films.

Different deposition techniques have been used to obtain Bi films, depending on the required thickness and final application; they include molecular beam epitaxy, evaporation, sputtering, pulsed laser ablation and electrodeposition [1,3,17,19-32]. Bi films deposited by physical techniques such as 
thermal evaporation and sputtering may result in small grain sizes and poor transport properties, and they may produce spectra with undesirable low energy tails when used as X-ray absorbers [26]. Besides, with these techniques it is difficult to obtain good quality films with thickness above $1 \mu \mathrm{m}$.

On the other hand, electrochemical deposition stands out due to its unique properties, i. e. simplicity, speed, ease of control, cost-effectiveness and the ability to deposit onto substrates with complex geometries [27-29]. Thus, electrodeposited bismuth layers display clear advantages. In fact, electrodeposition is the most suitable technique for films several microns thick, with deposition rates in the order of $0.2 \mu \mathrm{m} / \mathrm{min}$ reported [5,8,30].

It is widely accepted how changing the parameters of electrochemical deposition such as substrate, electrolyte composition, $\mathrm{pH}$ and concentration, deposition potential or current has a clear influence in the resulting coating morphology, and hence in its physical properties. In the case of $\mathrm{Bi}$, Zhu et al. [33] describe how the crystal orientation and melting point of electrodeposited $\mathrm{Bi}$ nanowires is highly influenced by the applied potential. Jiang et al. [31] focus on the importance of the deposition substrate, and several other studies are devoted to the importance of the additives, hence electrolyte composition [32]. Finally, other studies analyse the effect of deposition substrate and potential [34]. However little has been mentioned concerning the comparison between deposition methods with dynamic or static potential, or combined with other conditions such as additives, stirring or temperature changes, and their effects on transport properties. Static potential deposition is typically used when we are interested in adjusting a potential in order to prevent possible side reactions which may generate impurities or gas products [35], while constant current deposition usually guarantees a constant crystal growth and large crystals, when a single process may occur [36]. Dynamic potential methods, on the other hand, are normally used when the conditions of static deposition may not be optimal, and they have the advantage that the reversal scan eliminates the part of the deposit which 
has not been well adhered [37], which in turn renders final films with higher purity and quality or compacity.

This work studies the electrodeposition of bismuth using different solutions and methods in an attempt to further improve the physical properties of Bi coatings several $\mu$ m-thick, especially the electric transport at low temperatures. Moreover, it establishes an empirical correlation of electrodeposition parameters (electrolyte, the deposition method, the temperature and the presence of stirring) with morphology and electrical resistivity of the films. Two different electrolytes have been used, the first containing only bismuth nitrate and nitric acid, and the second containing the mentioned compounds plus other additives such as glycerol, tartaric acid and potassium nitrate. The resulting films reported are all pure and display high crystal quality in all cases, something that is not so usual [21]. Also, through a selection of variables during electrodeposition, it is concluded that using different electrolytes has a clear influence on the resulting properties of the films and on the best deposition method (static or dynamic). Additives have a determinant role, both in the morphology and the resistivity. A clear correlation is found between grain size and resistivity of the films, with better resistivity (lower values and better temperature dependence) when the grain size increases. Also, larger in-plane grain sizes are correlated in general to lower surface roughness. As a conclusion, the best films are found to display resistivity ratios close to unity and minimal resistivity values close to those of bulk bismuth, and are obtained using the electrolyte with additives and following a dynamic deposition and heating, although the effect of temperature is a second order parameter in this case.

\section{Experimental}

\subsection{Electrodeposition}


Bismuth thin films were prepared by electrochemical deposition on gold coated glass. Au was deposited by thermal evaporation up to a $10 \mathrm{~nm}$ thickness on soda lime glass $(24 \mathrm{~mm} \times 70 \mathrm{~mm}$ pieces) coated previously with a $\operatorname{Cr}(2 \mathrm{~nm})$ adhesion layer obtained also by thermal evaporation. The roughness of the final Au surface is $1 \mathrm{~nm}$ as measured with an Atomic force microscopy equipment (AFM; Agilent Technologies, model5400 SPM). The $10 \mathrm{~nm}$ thickness of the Au layers was chosen in order to ensure a homogeneous continuous film while minimizing the Au contribution in resistance measurements, because of the large difference between $\mathrm{Bi}$ and Au resistivities, and thus facilitating the measurements of Bi films resistance.

As shown in Table 1, two different aqueous solutions, purged for at least 10 min with Argon to remove ambient oxygen, were tested as electrolyte: 1) $0.01 \mathrm{M}$ bismuth nitrate pentahydrate $\left(\mathrm{BiNO}_{3} \cdot 5 \mathrm{H}_{2} \mathrm{O}\right.$, ACS Reagent, $\geq 98 \%$, Sigma-Aldrich) and $1 \mathrm{M}$ nitric acid $\left(\mathrm{HNO}_{3}, \mathrm{ACS}\right.$ Reagent, 70\%, Sigma-Aldrich); 2) $0.15 \mathrm{M}$ bismuth nitrate pentahydrate $\left(\mathrm{BiNO}_{3} \cdot 5 \mathrm{H}_{2} \mathrm{O}\right.$, ACS Reagent, $\geq 98 \%$, Sigma-Aldrich), 1.35 M glycerol (ACS Reagent, $\geq 99.5 \%$, Sigma-Aldrich), 1.15 M potassium hydroxide (KOH, ACS Reagent, $\geq 85 \%$, Sigma-Aldrich), 0.33 M DL-tartaric acid (Reagent plus ${ }^{\circledR}$, 99\%, Aldrich), and $16 \mathrm{M}$ nitric acid was added up to a pH of 0 [28].

A VMP model potentiostat (Biologic) was used for electrodeposition control, using a three-electrode cell configuration containing $35 \mathrm{ml}$ electrolyte solution. A Pt foil (Goodfellow 99.99\%, $5 \mathrm{~cm}^{2}$ exposure) was used as counter electrode and a $\mathrm{Ag} / \mathrm{AgCl}$ (sat $\mathrm{KCl}$ ) as reference electrode (Bioanalytical systems, BAS). The working electrode, where coatings were deposited, was the Au coated soda-lime glass. Both working and counter electrode are placed at a fixed $1 \mathrm{~cm}$ distance using two Teflon pieces on top and bottom of the Au coated glass piece described above. The bottom Teflon piece has an open space to allow diffusion of stirred solution, upon magnetic stirring at 300 rpm. Two strategies were used for electrodeposition: 1) Constant potential deposition at a potential of $-0.25 \mathrm{~V}$ vs $\mathrm{Ag} / \mathrm{AgCl}$, and 2) Dynamic potential deposition from open circuit potential to $-0.25 \mathrm{~V}$ and then back to $0 \mathrm{~V}$ vs $\mathrm{Ag} / \mathrm{AgCl}$, repeating several cycles up to a desired total charge, and at a speed 
of $10 \mathrm{mV} / \mathrm{s}$. Also, the effects of moderate stirring $(300 \mathrm{rpm})$ and heating $\left(55^{\circ} \mathrm{C}\right)$ on the properties of the films were evaluated. In all cases, and in order to compare and optimize films, the delivered charge was intended to yield thicknesses around 6 microns, with a certain variability, since these are the values suitable for high absorption efficiency (above 90\%) of X-rays with $\mathrm{E}<10 \mathrm{keV}$ [17-19]. Also, resistivity measurements of films synthesized with identical conditions and thickness varying between 5 and $8 \mu \mathrm{m}$ have revealed that the $\rho(T)$ of the films is independent of thickness, in this range.

\subsection{Morphological, chemical and electrical characterization}

Surface topography, grain size and thickness were determined from Scanning Electron Microscopy (SEM) images of the samples using a Quanta FEI 200 FEG-ESEM instrument). Typical operating parameters were $20-30 \mathrm{KeV}$ accelerating voltage and 2.5-3.0 $\mathrm{nm}$ spot size. SEM planar images of a surface area of $900 \mu \mathrm{m}^{2}$ were processed and analyzed using Mountains Map Premium software (Digital Surf Co.), in order to evaluate average grain size and surface roughness. Thickness was measured using the same conditions, from transversal SEM images, and correlated with charge used for deposition in every experiment.

The crystal structure and eventual preferential orientations of the coatings were analyzed by X-ray diffraction (XRD), using a Siemens D500 diffractometer, with $\mathrm{Cu}$ K $\alpha$ radiation; $\theta-2 \theta$ scans were carried out varying $2 \theta$ between $20^{\circ}$ and $80^{\circ}$, with $0.02^{\circ}$ steps. X-ray photoelectron spectroscopy (XPS) measurements were performed at room temperature using an AXIS Ultra from Kratos hemispherical analyzer with monochromatic $\mathrm{Al} \mathrm{KCu}_{\alpha}$ radiation, in order to investigate the composition of the films surface.

Resistance of the films was measured from room temperature down to $2 \mathrm{~K}$ in a Quantum Design PPMS, using the standard 4-probe technique: 4 parallel $\mathrm{Au}$ pads were evaporated on the films, extending on the substrate close to them, with the leads being micro-contacted on the pads. Because 
of the large difference between the resistivities of $\mathrm{Bi}$ and $\mathrm{Au}$, it is very important a careful subtraction of the contribution of the Au seed layer (required for electrodeposition) to the measured resistance. For this, it is crucial to insure that the Au layer resistance is significantly smaller than that of the $\mathrm{Bi}$ film; this was guaranteed by using a thin enough Au seed layer (10nm), measuring its resistance in the same geometry as that of Bi films, and then subtracting it to the measured resistance of Bi samples by assuming a circuit of two resistances in parallel corresponding respectively to the $\mathrm{Au}$ and Bi layers. For the best Bi films (lowest resistance) the Au layer contribution amounts at most to $3-4 \%$ of the total at low temperatures; as the Bi resistance increases, the Au contribution to the measured resistance is increasingly important, and so its correction (difference between the measured resistance and the resistance of the $\mathrm{Bi}$ film) also increases; in the worst cases (highest measured resistances) this correction amounts to $15 \%$. Since most Bi films display semiconductor behaviour, this also implies that the resistance ratio (R300/R2) values, where $R 300 / R 2 \equiv R(300 K) / R(2 K)$, are affected by this correction; this effect is below $10 \%$ in the most unfavourable cases.

\section{Results}

\subsection{Electrochemical deposition of bismuth}

Cyclic voltammetries of the solutions run at $10 \mathrm{mV} / \mathrm{s}$, and starting at the respective rest potentials, show several electrochemical features of $\mathrm{Bi}$ deposition that agree with those described elsewhere $[1,38]$. The electrochemical behavior in both cases and for the two electrolytes used is shown in figures 1 and 2 respectively. It may be observed that both solutions display the same I vs t behavior for both methods, and differ only in the fact that solution 2 reaches higher current density values than the process with solution 1 , for the same applied potentials, [38]. The initial times of the deposition slightly differ for both cases (figure 2), which may be due to the use of a different electrolyte.

In both cases, an electrochemical wave is observed at potentials around $0.2 \mathrm{~V}$ vs $\mathrm{Ag} / \mathrm{AgCl}$, interpreted as Bi underpotential deposition (UPD). Furthermore, a wide wave for extended Bi 
deposition is observed starting near $0 \mathrm{~V}$ vs $\mathrm{Ag} / \mathrm{AgCl}$ for both solutions used, with a ramp shape that indicates a resistive behavior due to the deposited coating (figure 1) [1,39]. There is no evidence of hydrogen evolution, in agreement with literature [40].

The electrodeposition has been performed by two methods: using constant potential or dynamic potential sweeps, as described in Section 2.1. After analyzing the effect of the electrolyte and the deposition method, which turned out to be the most influential parameters as we shall see, and in search of further improvement of the coatings, we also studied the effect of stirring (only in solution 1), heating the solution (up to $55^{\circ} \mathrm{C}$ ), plus warming and stirring simultaneously, on the electrochemical behavior of the deposition. Stirring could not be performed with solution 2 since this solution favored the formation of solid $\mathrm{Bi}^{+3}$ species [41] that disturbed the quality of the Bi film upon stirring. Representative samples of all the mentioned conditions are shown in Table 1, along with further characterization parameters.

From Table 1 it is clear that stirring increases the current density, as expected from a larger concentration gradient at the double layer, magnitude which controls the current density [42]. High temperature does not have such a remarkable influence on the deposition current density although current increases due to an increased diffusion of metal ion from the electrolyte to the cathode [43]; on the other hand, if both strategies are used simultaneously a larger influence of stirring is observed, with a similar current $\left(\mathrm{mA} / \mathrm{cm}^{2}\right)$ behavior.

All electrodeposited films show a white-grey color, as expected for $\mathrm{Bi}$, and are uniform to the naked eye. For an area of $5 \mathrm{~cm}^{2}$, all deposited layers require a charge in the range of 5.6 to $8.4 \mathrm{C} / \mu \mathrm{m}$. Dynamic deposition or heating increase the required charge for a certain thickness, as expected. All deposition processes have similar rates, and are comparable to the reported $0.2 \mu \mathrm{m} / \mathrm{min}[5,8,30]$, with slightly superior values upon heating or stirring. 


\subsection{Structural and microstructural characterization}

In the search of pure, compact Bi coatings with uniform thickness and low roughness, and with grain shape and size that maximize low temperature electrical conduction, correlations have been studied between deposition parameters, morphology of the obtained layers, and their resistivity. The structure, microstructure and chemical composition at the surface have been studied by SEM, XRD and XPS, as described in Section 2.2.

X-ray diffraction patterns (Figure 3) from all the coatings agree with the pattern of polycrystalline and pure rhombohedric $\mathrm{Bi}$ [44], with no evidence of other crystalline phases or impurities. The very low width of the peaks, detected by the clear differentiation between $K_{\alpha 1}$ and $K_{\alpha 2}$ reflections (inset in Fig.3), is indicative of the high crystallinity of all films. Relative peak intensities, however, differ from those expected for fully polycrystalline bulk samples, and are dependent on the solution and deposition method used (Figure 4). All films display a significant reduction in intensity of the (012) reflection, the most intense in bulk polycrystalline $\mathrm{Bi}$ [45], suggesting some degree of preferential orientation, which depends on the deposition parameters used, and that could be expected given the anisotropy of the rhombohedral Bi crystal structure. While absolute changes of peak intensity are observed for the same thickness and the intensity ratios of the 5 main peaks change with deposition conditions (Fig.4), these intensity ratios do not have a straightforward correlation with the corresponding deposition conditions. The clearest result is the significant decrease of the intensity of peak (012) -the most intense in bulk- when using solution 1 in static mode. In the case of solution 2, although (012) is also lower in intensity, the relative peak intensities are closer to those of bulk $\mathrm{Bi}$, and are similar in the three studied samples.

XPS analysis was performed to know the composition of the bismuth films surface. All the analyzed samples only contain bismuth, carbon and oxygen, discarding other possible impurities derived from ambient atmosphere or solution (data not shown). 
Three of the samples were studied in detail using higher resolution for the Bi $4 \mathrm{f}$ XPS peak, with and without etching, to identify possible composition differences between films surface and the volume immediately below. The results evidence the existence of $\mathrm{Bi} 4 \mathrm{f}_{5 / 2}$ and $4 \mathrm{f}_{7 / 2}$ peaks corresponding to both oxidized bismuth species (158.6eV and $163.9 \mathrm{eV}$ corresponding respectively to $\mathrm{Bi} 4 \mathrm{f}_{7 / 2}$ and $\mathrm{Bi}$ $4 \mathrm{f}_{5 / 2}$ ), and to elemental $\mathrm{Bi}$ (binding energies $156.9 \mathrm{eV}\left(\mathrm{Bi} 4 \mathrm{f}_{7 / 2}\right)$ and $162.2 \mathrm{eV}\left(\mathrm{Bi} 4 \mathrm{f}_{5 / 2}\right)$ ) at the surface. Oxidation is also confirmed by the presence of oxygen $\mathrm{O} 1 \mathrm{~s}$ peaks. However, these oxygen $\mathrm{O} 1 \mathrm{~s}$ peaks disappear as we etch the surface of the film. In all samples studied, carbon and oxygen present in the surface practically disappear after etching with argon ions is performed during 120 seconds (Figure 5a), which corresponds to the approximate removal of a 10-20nm surface thickness. For two of the samples (D and F), the etching was continued for 300 seconds $(\approx 30-40 \mathrm{~nm})$, in which case carbon content resulted near $0 \%$ and oxygen content around 1\% (data not shown). The doublet signal expected for $\mathrm{Bi}, \mathrm{Bi} 4 \mathrm{f}_{5 / 2}$, and $\mathrm{Bi} 4 \mathrm{f}_{7 / 2}$ is observed splitted, due to the presence of oxidized $\mathrm{Bi}$ at the surface, as mentioned above (Figure 5a). However, the relative amount of elemental un-oxidized Bi signal is larger with increasing etching time, while the oxidized $\mathrm{Bi}$ signal decreases substantially [27]. This confirms that the films are mostly made of pure metallic bismuth, and states that only a small degree of oxidation occurs in ambient conditions (30 nm vs $6000 \mathrm{~nm}$ coating). The C 1s core level spectra reveals the usual signal of endemic carbon (Figure 5b). This carbon includes aliphatic carbon species (C1 peak at $\sim 284.8 \mathrm{eV}$ ) and species with $\mathrm{C}-\mathrm{O}$ bond at $286.0 \mathrm{eV}$ (C2 peak). Other species which may be related to hydroxycarbonate phases appear at $\sim 288.0 \mathrm{eV}$ (C3 peak). The sample F, made with solution 2, has the biggest peak at $\sim 286.0 \mathrm{eV}(\mathrm{C} 2)$, which indicates the presence of species with C-O bond, possibly related with the use of additives [46]. No $\mathrm{N}$ 1s peaks are observed, discarding possible nitrate contamination.

In the case of oxygen O 1s XPS signals, the observations agree with the existence of a certain degree of oxidation of the surface, as mentioned, but $\mathrm{O}$ 1s peak deconvolution (Figure $5 \mathrm{c}$ ) evidences that the oxidized phase is not a simple bismuth oxide. The $\mathrm{O} 1$ and $\mathrm{O} 2$ peaks evidence different types of 
oxygens, which could be related to various complex bismuth oxides [47], but the existence of a third peak at higher binding energies $(\sim 532 \mathrm{eV})$ entails the existence of species such as hydrated hydroxo $\left(\mathrm{OH}^{-} / \mathrm{H}_{2} \mathrm{O}\right)$, or hydroxycarbonate phases, also detected in the $\mathrm{C}$ 1s spectra analysis. Oxygenated species may come from the oxidized bismuth phase and also from adsorbed carbonated species, in both cases disappear upon etching, and are not observed in the measured X-ray diffraction pattern.

In general, samples synthesized with solution 2 reveal a larger amount of oxygenated species at the surface, which may be due to the presence of more additives in the electrolyte. However, if the XPS analysis is performed 10 months after preparation for the same samples, the ones made with solution 2 have not increased their amount of oxygenated species, while samples made with solution 1 have reached the same content than those from solution 2. This suggests a faster oxidation in samples obtained from solution 2 and also a maximum limiting level of oxidation of the films surface, with a thickness of 30-40 nm, since only $\mathrm{Bi}$ is observed after a $300 \mathrm{~s}$ etching. Hence the aging effect of the samples in atmospheric conditions is very limited in both cases.

The microstructure of electrodeposited Bi films has been studied by SEM (Fig.6). In general, all films display a similar morphology, exhibiting a granular topography with well-defined crystals and a clear tendency to anisotropic rhombohedral formations, in agreement with the rhombohedral $\mathrm{Bi}$ phase revealed by X-ray diffraction. However, the use of different synthesis conditions does have a significant influence on roughness and grain size of the films, as expected $[29,31,34,48]$.

Table 1 displays the resulting root mean square (RMS) roughness and grain sizes for the representative samples analyzed in this work, as extracted from analyses of the SEM images, (see section 2.2). With solution 1 a notably flat film is obtained in static mode stirring and heating. With solution 2, the roughness of all samples is very similar to this latter sample and also similar among all prepared with such solution, but the sample synthesized in dynamic mode and heating has the largest crystal size. 
Analyses of the possible relationships between grain sizes and the absolute and relative intensity of main peaks in the $\theta-2 \theta$ X-ray diffraction patterns have also been performed, since a variation in relative intensity is observed when changing the deposition parameters, as described above. However, no clear correlations have been found between the average grain size and the intensity ratios of any pair of the 5 most intense peaks in bulk. All samples have crystal sizes around 3 microns, and they have the peak (012) depressed, particularly films from solution 1, evidencing a degree of orientation. However, no clear correlation of orientation with grain size is detected.

\subsection{Resistivity}

The evaluation of the functional properties of the Bi films, as a probe for optimization of the electrodeposition process, has been performed by measuring the electrical resistivity which, in view of the Wiedemann-Franz law [17], can be used as a proxy of thermal conductivity. This is a crucial parameter, since $\mathrm{Bi}$ coatings require high thermal conductivity, in order to be used as X-ray absorbers.

$\mathrm{R}(\mathrm{T})$ measurements have been performed as described in Section 2.2. They have been found very reproducible, and without aging effects in the span of several months (samples $\mathrm{C}$ and $\mathrm{H}$ were measured after 3 months and the results were coincident), indicating that the slight surface oxidation observed by XPS has no effect on electrical transport in the coatings. Fig. 7 shows the temperature dependence of the electrical resistivity of the films listed in Table 1 . Both the temperature dependence and the values vary significantly among samples; clearly, the deposition parameters and methods affect the electrical transport, and therefore also the thermal transport.

While bulk $\mathrm{Bi}$ is a semimetal (that is, displays metallic behaviour but with resistivity much higher than metals, due to the low charge carrier density), it is well known that Bi films display most often semiconducting behaviour $[3,8,17,19,22,23]$; quite often also this semiconducting behaviour flattens 
and saturates at low temperature. The exact shape of the $\mathrm{R}(\mathrm{T})$ films appears to depend on their thickness and the deposition technique; this variability has been ascribed to the peculiar band structure of bismuth, responsible of its semi-metallic bulk behaviour (highly anisotropic Fermi surface, low carrier concentrations, small effective carrier masses and high carrier mobilities), which makes the material extremely sensitive to defects $[3,23]$. It has also been observed that, depending on the type of defects and film thickness, the semiconductor behaviour can be smoothened, and metallic behaviour can be recovered at high enough temperatures. On the other hand, a metallic dependence has also been obtained after thermal annealing of the films $[5,19]$. Thinking in those terms, the resistance ratio $R_{300} / R_{2}=R(300 K) / R(2 K)$ can be used to describe the flatness of the $R(T)$, i.e. whether the films are more semiconducting-like or closer to metallic behaviour. As reference, $R_{300} / R_{2}$ of films with thickness in the order of microns have been reported with values as low as 0.2 and up to 1 in the literature $[19,20]$.

The resistivities of the films reported in this work display metallic behaviour from room temperature down to a temperature $T_{\min }$ characteristic of each sample; at $\mathrm{T}<\mathrm{T}_{\min }$ the resistance displays semiconducting behaviour, tending to saturate at the lower temperatures ( $T<40 \mathrm{~K})$. The extent of the metallic behaviour can be evaluated from $T_{\min }$ : the lower $T_{\min }$, the more extense temperature window with metallic $R(T)$ and consequently the higher $R_{300} / R_{2}$.

The deposition method and solution affect the values of the resistivity and also its temperature dependence, parametrized with $\mathrm{R}_{300} / \mathrm{R}_{2}$ and $\mathrm{T}_{\min }$. It is found that films with lower resistivity (at both room temperature and the lowest measured temperature) display higher $R_{300} / R_{2}$ values, as shown in Fig. 8a. In addition, a correlation between the resistivity values and the average grain size is observed (Fig.8b): lower resistivities (and also higher $\mathrm{R}_{300} / \mathrm{R}_{2}$, according to Fig. $8 \mathrm{a}$ ) are associated to larger grain sizes, which can be at least partially understood because of the smaller number of grain boundaries. 
Moreover, the films obtained from solution 2 (F, G, H) display lower $T_{\min }$ values than those from solution 1. Since it has been found (see inset in Figure 4) that these films have the main XRD peak (012) more intense, it follows that better $\mathrm{R}(\mathrm{T})$ behaviour (more metallic, more bulk-like) is related to diffraction patterns also closer to bulk; in fact, when plotting $T_{\min }$ and $R_{300} / R_{2}$ as a function of the intensity of peak (012) two data groups, corresponding respectively to films from solution 1 and 2, can be clearly differentiated (Fig.9).

As stated above, Bi films to be used as absorbers require low resistance values at low temperatures. Thus, films with $\mathrm{R}_{300} / \mathrm{R}_{2} \sim 1$ and lower resistivity are close to the desired -optimal- performances. Fig.7 evidences how crucial is the solution chosen and the deposition method used: the worst $\rho(\mathrm{T})$ correspond to films made by solution 1 in dynamic mode, and by solution 2 in static mode; Fig. 9 also reveals that the best $\rho(T)$ of films from solution 2 are significantly better than the best ones from solution 1 , in the sense that they display better resistance ratios $R_{300} / R_{2}$ and also better resistivity values, both at room temperatures and at low temperatures. In fact the best film obtained with solution 1 has a resistivity at $2 \mathrm{~K}$ nearly twice as high as the best film obtained with solution 2 . Thus, the best conditions correspond to solution 2 in dynamic mode and heating: in this case, $R_{300} / R_{2}=1$ is obtained, with a lowest resistivity value of $160 \mu \mathrm{cm}$ obtained at $150 \mathrm{~K}$ and a low temperature saturation value of $\sim 200 \mu \Omega \mathrm{cm}$.

\section{Conclusions}

The observations described suggest that the best films, following a criterion of resistivity, are those obtained from the electrolyte 2 using dynamic potential deposition; while heating and stirring have proven less crucial. These films display metallic behaviour down to $\mathrm{T} \sim 150 \mathrm{~K}$; at lower temperatures resistivity smoothly increases, saturating at $\mathrm{T}<\sim 40-20 \mathrm{~K}$ to a value close to the room temperature, thus providing $\mathrm{R}_{300} / \mathrm{R}_{2} \sim 1$. The low temperature resistivity values of the optimal films are $\sim 0.16-0.2$ $\mathrm{m} \Omega \mathrm{cm}$, similar or slightly below the best reported values for Bi films of similar thicknesses, also 
electrodeposited [8,19], while the room temperature value for bulk $\mathrm{Bi}$ is $0.13 \mathrm{~m} \Omega \mathrm{cm}$. An improvement of $R_{300} / R_{2}$ and resistivity values for films annealed close to the Bi melting point has been reported [5,19], but this kind of annealing is not suitable for their application as absorbers in radiation detectors, since it could not be borne by the devices on which the Bi layers must be deposited. Therefore, the results shown are similar to the best found in literature without annealing.

Summarizing, the electrodeposition processes have been optimized to get Bi films suitable for use as X-ray absorbers. The effects of different deposition parameters and techniques, such as electrolyte composition, dynamic or static potential deposition, heating and stirring are analysed in terms of films purity, crystallinity, morphology and resistivity. Correlations between roughness, grain size and resistivity behaviour allowed to conclude that electrolyte composition and deposition method are the most important variables to optimize the films in terms of morphology and electronic transport; it is also observed that the deposition method providing best films depends on the electrolyte.

The optimal films are obtained with the solution containing additives such tartaric acid and glycerol, and using the dynamic potential method. These films display metallic $\rho(T)$ curves from room temperature down to $\sim 150 \mathrm{~K}$; at lower temperatures the resistivity smoothly increases before saturating at $\mathrm{T}<20-40 \mathrm{~K}$; this behaviour provides resistance ratios $\mathrm{R}_{300} / \mathrm{R}_{2} \sim 1$ and with the lowest resistivity values close to the bulk ones, similarly to those reported so far for the best $\mathrm{Bi}$ films of similar thickness and without annealing.

\section{Acknowledgements}

Work financed by the Spanish Ministerio de Economía y Competitividad-MINECO (projects ESP2014-53672-C3-2-P , ESP2016-76683-C3-2-R and ESP2014-59309-JIN), the European Space 
Agency-ESA (CTP Contract “Optimization of a European TES array”) and the European Commission (H2020 project AHEAD: “Integrated activities for the high energy astrophysics domain”). Personnel from ICMAB acknowledge financial support from MINECO, through the “Severo Ochoa” Programme for Centres of Excellence in R\&D (SEV- 2015-0496). RMJ wishes to thank MINECO for her FPI contract. We acknowledge also the help of Guillermo Antorrena (INA) on XPS data.

\section{REFERENCES}

[1] B. O’Brien, M. Plaza, L.Y. Zhu, L. Perez, C.L. Chien, P.C. Searson, Magneto transport properties of electrodeposited bismuth films, J. Phys. Chem. C 112 (2008) 12018-12023.

[2] O. Prakash, A. Kumar, A. Thamizhavel, S. Ramakrishnan, Evidence for bulk superconductivity in pure bismuth single crystals at ambient pressure, Science 52 (2017) 52-55.

[3] C. A. Hoffman, J.R. Meyer, F.J. Bartoli, A. Di Venere, X.J. Yi, C.L. Hou, H.C. Wang, J.B. Ketterson, Semimetal-to-semiconductor transition in bismuth thin films, Phys. Rev. B 48 (1993) 11431-11434.

[4] M. Lu, R.J. Zieve, A. van Hulst, H.M. Jaeger, T.F. Rosenbaum, S. Radelaar, Low-temperature electrical-transport properties of single-crystal bismuth films under pressure, Phys. Rev. B 53 (1996) 1609-1615.

[5] F.Y. Yang, K. Liu, C.L. Chien, P.C. Searson, Large magnetoresistance and finite-Size effects in electrodeposited single-crystal Bi thin films, Phys. Rev. Lett. 82 (1999) 3328-3331.

[6] H. Du, X. Sun, X. Liu, X. Wu, J. Wang, M. Tian, A. Zhao, Y. Luo, J. Yang, B. Wang, J.G. Hou, Surface Landau levels and spin states in bismuth (111) ultrathin films, Nature Comm. 7 (2016) 10814.

[7] L. Li, Y. Zhang, G. Li, L. Zhang, A route to fabricate single crystalline bismuth nanowire arrays with different diameters, Chem. Phys. Lett. 378 (2003) 244-249. 
[8] F.Y. Yang, K. Liu, K. Hong, D.H. Reich, P.C. Searson, C.L. Chien, Large magnetoresistance of electrodeposited single-crystal bismuth thin films, Science 284 (1999) 1335-1337.

[9] J.P. Ziegler, Status of reversible electrodeposition electrochromic devices, Solar Energ. Mater. \& Solar Cells 56 (1999) 477-493.

[10] S.I. Cordoba de Torresi, I.A. Carlos, Optical characterization of bismuth reversible electrodeposition, J. Electroanal. Chem. 414 (1996) 11-16.

[11] J.D.Yao, J.M. Shao, G.W. Yang, Ultra-broadband and high-responsive photodetectors based on bismuth film at room temperature, Scientific Reports 5 (2015) 12320.

[12] N.Srimathy, A.R.Kumar, Structural and optical characterization of thermally evaporated bismuth and antimony films for photovoltaic applications, Superlattices and Microstructures 93 (2016) 1-10.

[13] Y. Lin, X. Sun, M.S. Dresselhaus, Theoretical investigation of thermoelectric transport properties of cylindrical Bi nanowires, Phys. Rev. B 62 (2000) 4610-4623.

[14] J. Ping, Y. Wang, J. Wu, Y. Ying, Development of an electrochemically reduced graphene oxide modified disposable bismuth film electrode and its application for stripping analysis of heavy metals in milk, Food Chemistry 151 (2014) 65-71.

[15] V. Rehacek, I. Hotovy, M. Vojs, Bismuth film voltammetric sensor on pyrolyzed photoresist/alumina support for determination of heavy metals, Electroanalysis 26 (2014) 898-903. [16] G.D. Pierini, M.F. Pistonesi, M.S. Di Nezio, M.E. Centurion, A pencil-lead bismuth film electrode and chemometric tools for simultaneous determination of heavy metals in propolis samples, Microchem. J. 125 (2015) 266-272.

[17] N. Iyomoto, J.E. Sadleir, E. Figueroa-Feliciano, T. Saab, S.R. Bandler, C.A. Kilbourne, J.A. Chervenak, D. Talley, F.M. Finkbeiner, R.P. Brekosky, M.A. Lindeman, R.L. Kelley, F.S. Porter, K.R. Boyce, Optimization of x-ray absorbers for TES microcalorimeters, Proc. SPIE 5501 (2014). 
[18] H. Kudo, T. Arakawa, S. Ohtsuka, T. Izumi, S. Shoji, H. Sato, H. Kobayashi, K. Mori, T.

Homma, T. Osaka, N. Iyomoto, R. Fujimoto, K. Mitsuda, N.Y. Yamasaki, T. Oshima, K. Futamoto, Y. Takei, T. Ichitsubo, T. Fujimori, Y. Ishisaki, U. Morita, T. Koga, K. Shinozaki, K. Sato, T.

Ohashi, Y. Kuroda, M. Onishi, K. Otake, F. Beppu, High sensitive X-ray microcalorimeter using Bi-Au microabsorber for imaging applications, Jpn. J. Appl. Phys. 43 (2004) 1190.

[19] A.D. Brown, S.R. Bandler, R. Brekosky , J.A. Chervenak, E. Figueroa-Feliciano, F. Finkbeiner , N. Iyomoto, R.L. Kelley , C.A. Kilbourne,F.S. Porter, S. Smith, T. Saab, J. Sadleir, Absorber materials for transition-edge sensor X-ray microcalorimeters, J. Low Temp. Phys. 151 (2008) 413417.

[20] M.P. Bruijn, M.L. Ridder, E. Krouwer, H.F.C. Hoevers, P.A.J. de Korte, J. van der Kuur, Progress in fabrication of microcalorimeter arrays: X-ray absorbers and high-density stripline wiring, Nuclear Instruments and Methods in Physics Research A 559 (2006) 444-446.

[21] L.M. Gades, T. W. Cecil, R. Divan, D.R. Schmidt, J.N. Ullom, T.J. Madden, A. Miceli, Development of Thick Electroplated Bismuth Absorbers for Large Collection Area Hard X-ray Transition Edge Sensors, IEEE Transactions on Applied Superconductivity 27(4) (2017). [22] T. Yoshino, K. Yoshida, T. Hagihara, K. Sato, U. Morita, Y. Ishisaki, R. Fujimoto, N.Y. Yamasaki, K. Mitsuda, Properties of vacuum-evaporated bismuth absorber, Nuclear Instruments and Methods in Physics Research A 559 (2006) 432-435.

[23] C.Grant Padwick, Resistivity measurements of thin films of bismuth: applications for building bolometric detectors, Master's Thesis, University of British Columbia (1997).

[24] B.Y. Jin, H.K. Wong, G.K. Wong, J.B. Ketterson, Effect of annealing on the transport properties of an epitaxial film of bismuth, Thin Solid Films 110 (1983) 29-36.

[25] S. Cho, Y. Kim, A.J. Freeman, G.K.L. Wong, J.B. Ketterson, L.J. Olafsen, I. Vurgaftman, J.R. Meyer, C.A. Hoffman, Large magnetoresistance in postannealed Bi thin films, Appl. Phys. Lett. 79 (2001) 3651-3653. 
[26] D. Yan, R. Divan, L.M. Gades, P. Kenesei, T.J. Madden, A. Miceli, J. Park, U.M. Patel, O. Quaranta, H. Sharma, D.A. Bennett, W.B. Doriese, J.W. Fowler, J.D. Gard, J.P. Hays-Wehle, K.M. Morgan, D. R. Schmidt, D.S. Swetz, J.N. Ullom, Eliminating the non-Gaussian spectral response of X-ray absorbers for transition-edge sensors, Appl. Phys. Lett. 111 (2017) 192602.

[27] A.R. Rajamani, S. Jothi, M.D. Kumar, S. Srikaanth, M.K. Singh, G. Otero-Irurueta, D. Ramasamy, M. Datta, M. Rangarajan, Effects of additives on kinetics morphologies and lead sensing property of electrodeposited bismuth films. J. Phys. Chem. C 120 (2016), 22398-22406.

[28] K. Liu, C.L. Chien, P.C. Searson, K. Yu-Zhang, Structural and magneto-transport properties of electrodeposited bismuth nanowires, Appl. Phys. Lett. 73 (1998) 1436-1438.

[29] C. Agapescu, A. Cojocaru, A. Cotarta, T. Visan, Electrodeposition of bismuth, tellurium, and bismuth telluride thin films from choline chloride-oxalic acid ionic liquid, J. Appl. Electrochem. 43 (2013) 309-321.

[30] C.L. Chen, F.Y. Yang, K. Liu, D.H. Reich, P.C. Searson. Very large magnetoresistance in electrodeposited single-crystal Bi thin films, J. Appl. Phys. 87 (2000) 4659-4664.

[31] S. Jiang, Y. Huang, F. Lao, N. Du, C. Yan, Synthesis of bismuth with various morphologies by electrodeposition, Inorg. Chem. Comm. 6 (2003) 781-785

[32] D.I. Tishkevich, S.S. Grabchikov, L.S. Tsybulskaya, V.S. Shendyukov, S.S. Perevoznikov, S.V. Trukhanov, E.L. Trukhanova, A.V. Trukhanov, D.A. Vinnik, Electrochemical deposition regimes and critical influence of organic additives on the structure of Bi films. Journal of alloys and compounds 735 (2017) 1943-1948.

[33] Y. Zhu, X. Dou, X. Huang, L. Li, G. Li, Thermal properties of Bi nanowire arrays with different orientations and diameters, J. Phys. Chem. B 110 (2006) 26189-26193.

[34] D. Bilican, J. Fornell, J. Sort, E. Pellicer, Electrochemical synthesis of Bi particles: Tuning particle shape through substrate type within a narrow potential window. Materials 10 (2017) 43. 
[35] P. Zanello, Inorganic electrochemistry: theory, practice and application, The Royal Society of Chemistry, Cambridge, 2003.

[36] D. Pletcher and F.C. Walsh, Industrial Electrochemistry $2^{\text {nd }}$ ed., Springer, Netherlands, 1993. [37] V. Bagotsky, Fundamentals of electrochemistry $2^{\text {nd }}$ ed., John Wiley and sons inc., New-Jersey (USA), 2006.

[38] S.B. Sadale, P.S. Patil, Nucleation and growth of bismuth thin films onto fluorine-doped tin oxide-coated conducting glass substrates from nitrate solutions. Solid state ionics 167 (2004) 273283.

[39] S. Holvoet, P. Hornya, S. Turgeon, P. Chevallier, J. Pireaux, D. Mantovani, Characterization of film failures by bismuth electrodeposition- Application to thin deformed fluorocarbon films for stent applications. Electrochim. Acta 55 (2010) 1042-1050.

[40] E. Sandnes, M.E. Williams, U. Bertocci , M.D. Vaudin, G.R. Stafford, Electrodeposition of bismuth from nitric acid electrolyte, Electrochim. Acta 52 (2007) 6221-6228.

[41] Y.M. Yukhin, T.V. Daminova, L.I. Afonina, B.B. Bokhonov, O.A. Logutenko, A.I. Aparnev, K.Y. Mikhailov, T.A. Udalova, V.I. Evseenko, Synthesis of bismuths (III) compounds for medical applications, Chemistry for Sustainable Development 12 (2004) 395-401.

[42] Bard and Faulkner, Electrochemical methods, fundamental and applications, Wiley 2001

[43] F. Xiao, C. Hangarter, B. Yoo, Y. Rheema, K. Leec, N.V. Myung, Recent progress in electrodeposition of thermoelectric thin films and nanostructures, Electrochim. Acta 53 (2008) 81038117.

[44] Y. Shu, W. Hu, Z. Liu, G. Shen, B. Xu, Z. Zhao, J. He, Y. Wang, Y. Tian, D. Yu, Coexistence of multiple metastable polytypes in rhombohedral bismuth, Nature scientific reports 6 (2015) 20337. [45] ICDD card No. 00-044-1246 for Bi 
[46] J. Moral-Vico, S. Sanchez-Redondo, M.P. Lichtenstein, C. Suñol, N. Casañ-Pastor, Nanocomposites of iridium oxide and conducting polymers as electroactive phases in biological media, Acta Biomater. 10 (2014) 2177-2186.

[47] C. D. Wagner, WM. Riggs, L.E. Davis and J.F. Moulder, Handbook of X-Ray photoelectron spectroscopy, Perkin Elmer Corporation, Minnesota (USA), 1979

[48] H. Sato, T. Homma, H. Kudo, T. Izumi, T. Osaka, S. Shoji, Three- dimensional microfabrication process using Bi electrodeposition for a highly sensitive X-ray imaging sensor, J. Electroanal, Chem. 584 (2005) 28-33. 


\section{TABLES}

Table 1. List of selected synthesized samples: electrolyte and electrodeposition conditions used, and their obtained thickness, charge/thickness ratio, surface roughness (RMS), grain size, room temperature resistivity $\rho(300 \mathrm{~K})$ and resistance ratio $R_{300} / R_{2}$ values.

\begin{tabular}{|c|c|c|c|c|c|c|c|c|c|c|c|}
\hline SAMPLE & ELECTROLYTE & $\begin{array}{l}\text { DEPOSITION } \\
\text { POTENTIAL }\end{array}$ & STIRRING & HEATING & $\begin{array}{c}\text { MAXIMUM } \\
\text { CURRENT }^{1} \\
\left(\mathrm{~mA} / \mathrm{cm}^{2}\right)\end{array}$ & $\begin{array}{c}\text { THICKNESS } \\
(\mu \mathrm{m})\end{array}$ & $\begin{array}{c}\text { CHARGE/ } \\
\text { THICKNESS } \\
(\mathrm{C} / \mu \mathrm{m})\end{array}$ & $\begin{array}{c}\text { RMS } \\
\text { roughness } \\
(\mu \mathrm{m})\end{array}$ & $\begin{array}{c}\text { GRAIN } \\
\text { SIZE } \\
(\mu \mathrm{m})\end{array}$ & $\begin{array}{c}\rho(300 K) \\
(\mu \Omega \mathrm{m})\end{array}$ & $\mathrm{R}_{300} / \mathrm{R}_{2}$ \\
\hline A & Solution 1 & Constant & No & No & -2.0 & 6.6 & 6.5 & 0.30 & $3.0 \pm 0.4$ & 3.0 & 0.81 \\
\hline B & Solution 1 & Constant & Yes & No & -3.5 & 6.4 & 6.3 & 0.27 & $2.5 \pm 0.3$ & 4.8 & 0.63 \\
\hline C & Solution 1 & Constant & No & Yes & -1.8 & 7.8 & 7.3 & 0.25 & $3.4 \pm 0.4$ & 3.2 & 0.72 \\
\hline D & Solution 1 & Constant & Yes & Yes & -5.1 & 8.0 & 7.1 & 0.10 & $3.0 \pm 0.2$ & 3.6 & 0.77 \\
\hline $\mathrm{E}$ & Solution 1 & Dynamic & Yes & No & -5.4 & 5.1 & 8.3 & 0.25 & $2.2 \pm 0.2$ & 7.3 & 0.48 \\
\hline $\mathrm{F}$ & Solution 2 & Constant & No & No & -5.6 & 5.9 & 5.6 & 0.11 & $3.1 \pm 0.3$ & 7.4 & 0.67 \\
\hline G & Solution 2 & Dynamic & No & No & -2.8 & 6.4 & 7.4 & 0.14 & $3.0 \pm 0.3$ & 2.0 & 0.96 \\
\hline $\mathrm{H}$ & Solution 2 & Dynamic & No & Yes & -3.6 & 6.4 & 8.4 & 0.12 & $3.7 \pm 0.3$ & 2.6 & 1.03 \\
\hline
\end{tabular}

${ }^{1}$ This column reflects the values of current at the plateaus obtained for constant potential depositions, and the maximum current obtained for dynamic potential depositions. 


\section{FIGURE CAPTIONS}

Fig. 1. CV curves and simultaneous dynamic electrodeposition at $10 \mathrm{mV} / \mathrm{s}$ for: (a) solution 1: (sample A) and (b) solution 2: (sample F). (No stirring or warming performed).

Fig. 2. Chronoamperometric response for electrodeposition at constant $-0.25 \mathrm{~V}$ vs $\mathrm{Ag} / \mathrm{AgCl}$ using: (a) solution 1 and (b) solution 2. (No stirring or warming performed). The 50s first seconds of the deposition are expanded for both cases.

Fig. 3. X-ray diffraction patterns $(\theta-2 \theta)$ of selected films, showing only rhombohedral Bi diffraction peaks, and different relative intensities. Symbols correspond to same samples referenced in Table 1.

Fig. 4. Relative intensities of the main diffraction peaks of rhombohedral $\mathrm{Bi}$, with respect to the intensity of the (012) peak, the most intense peak in bulk rhombohedral Bi [45]. The intensity ratios labelled as "bulk" correspond to [45]. Inset: Comparison of absolute intensity for peak (012) for similar thicknesses.

Fig. 5. XPS spectra of: (a) A film Bi 4f spectra with different etching times, (b) C 1s core level spectra of Bi films A, D and F (no etching), and (c) O 1s core level spectra of Bi films A, D and F (no etching).

Fig. 6. SEM images of the bismuth films listed in table 1. For each sample a planar and a crosssectional image (above the planar one) are shown.

Fig. 7. Electrical resistivity of the analysed Bi electrodeposited films, as a function of temperature. The error bars are smaller than the symbol.

Fig. 8. (a) Resistance ratio $\left(\mathrm{R}_{300} / \mathrm{R}_{2}\right)$ as a function of room temperature resistivity. (b) Resistance ratio $R_{300} / R_{2}$ as a function of the average grain size for the films displayed in Table 1 . Solid lines are only a guide to the eye. 
Fig. 9. Temperature $T_{\min }$ at which the minimum in $R(T)$ occurs, and resistance ratio $R_{300} / R_{2}$ (inset), as a function of the intensity of XRD peak (012). 


\section{FIGURES}

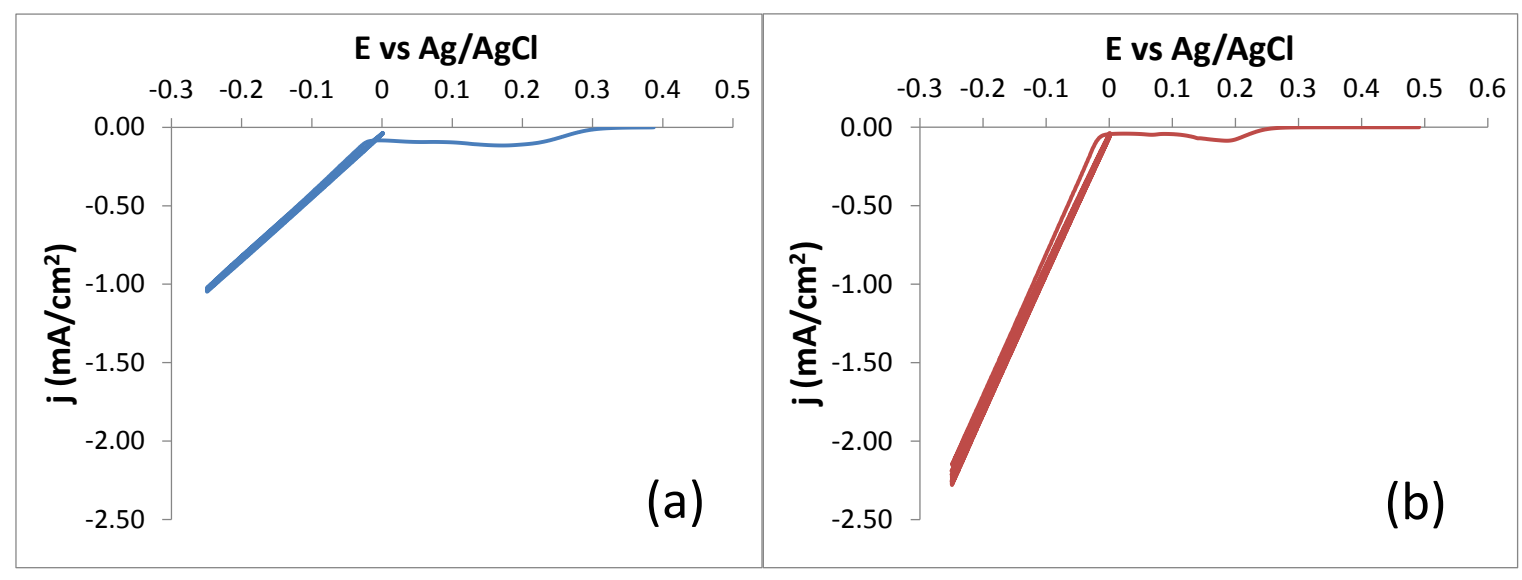

Fig. 1

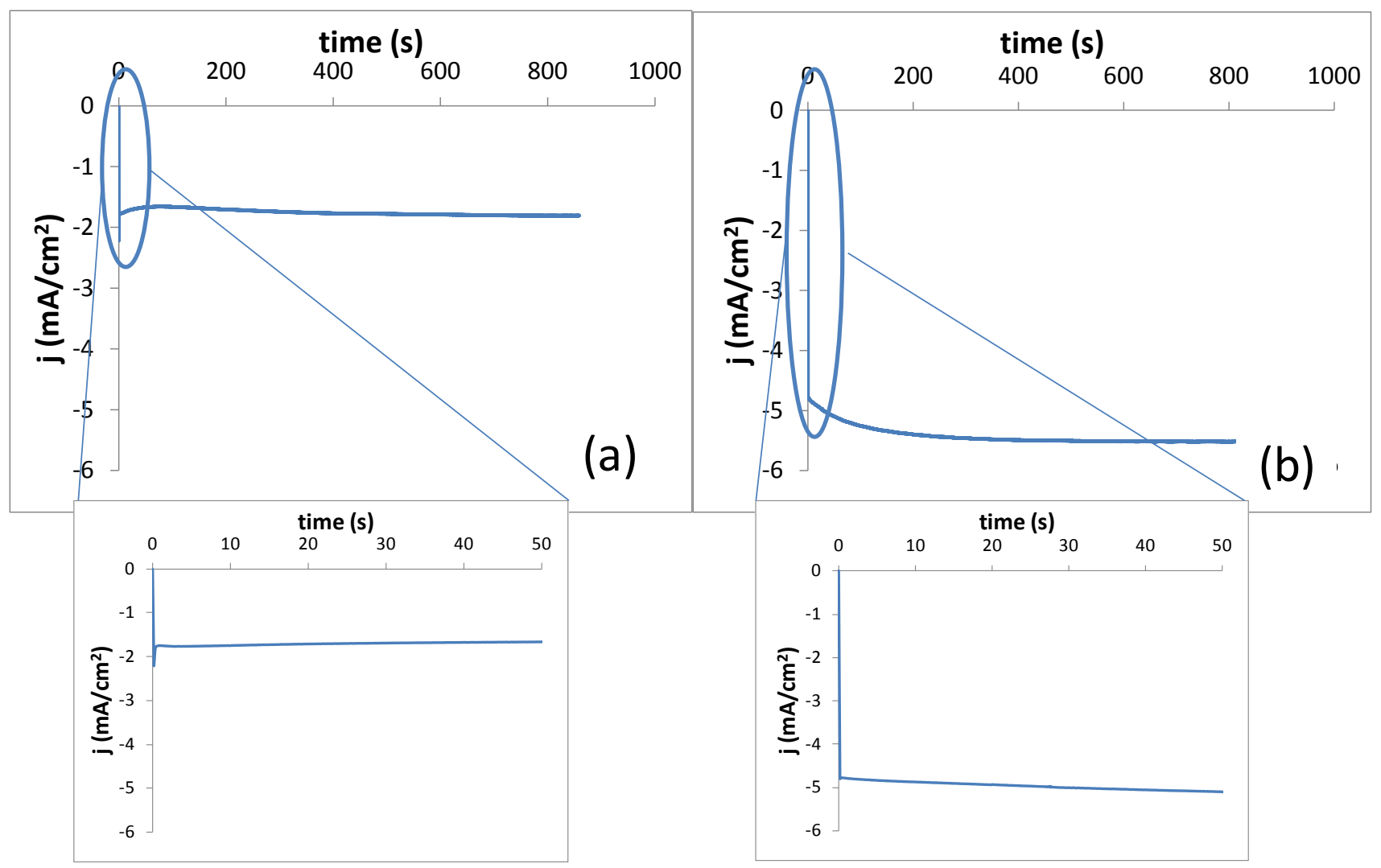

Fig. 2 


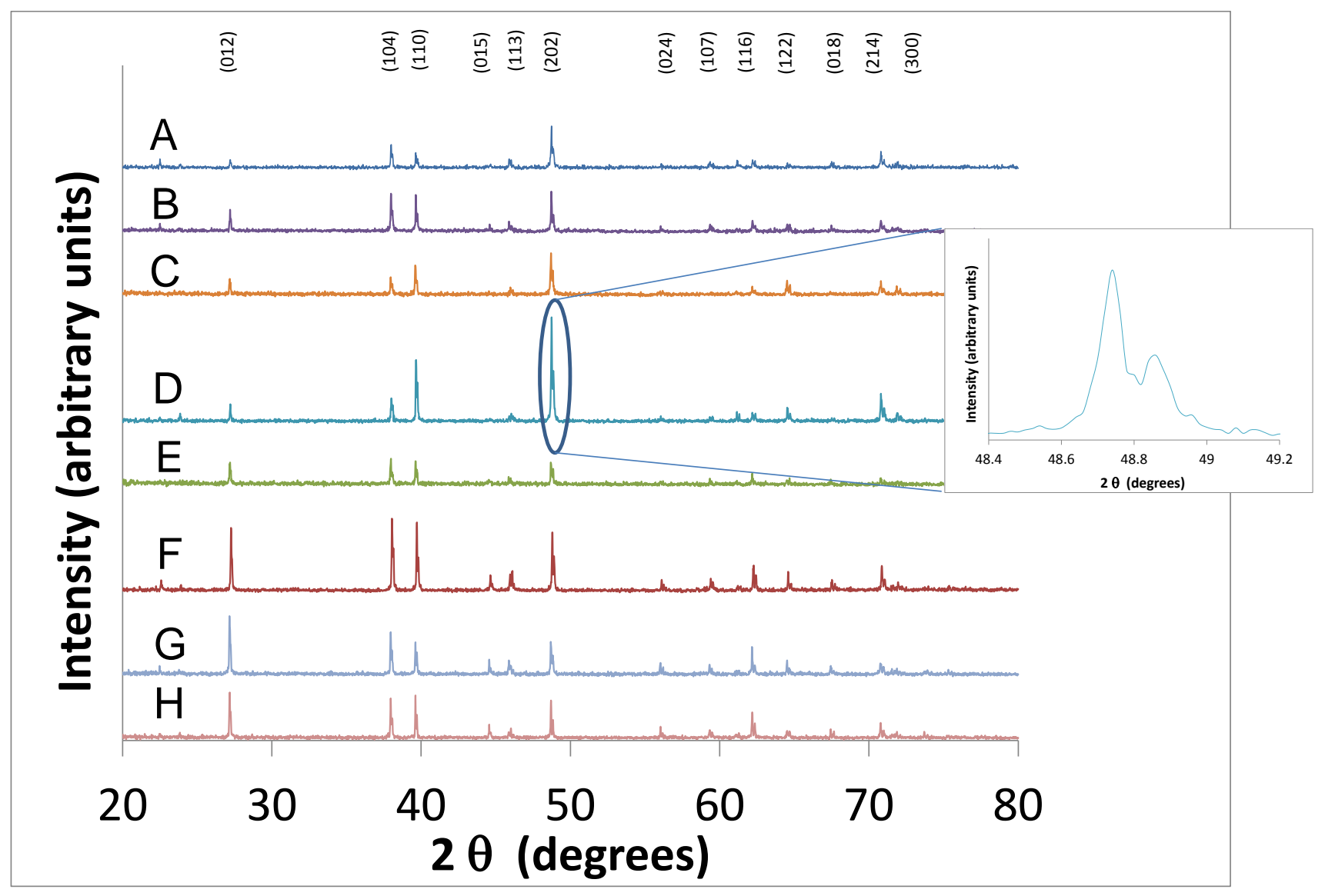

Fig. 3 


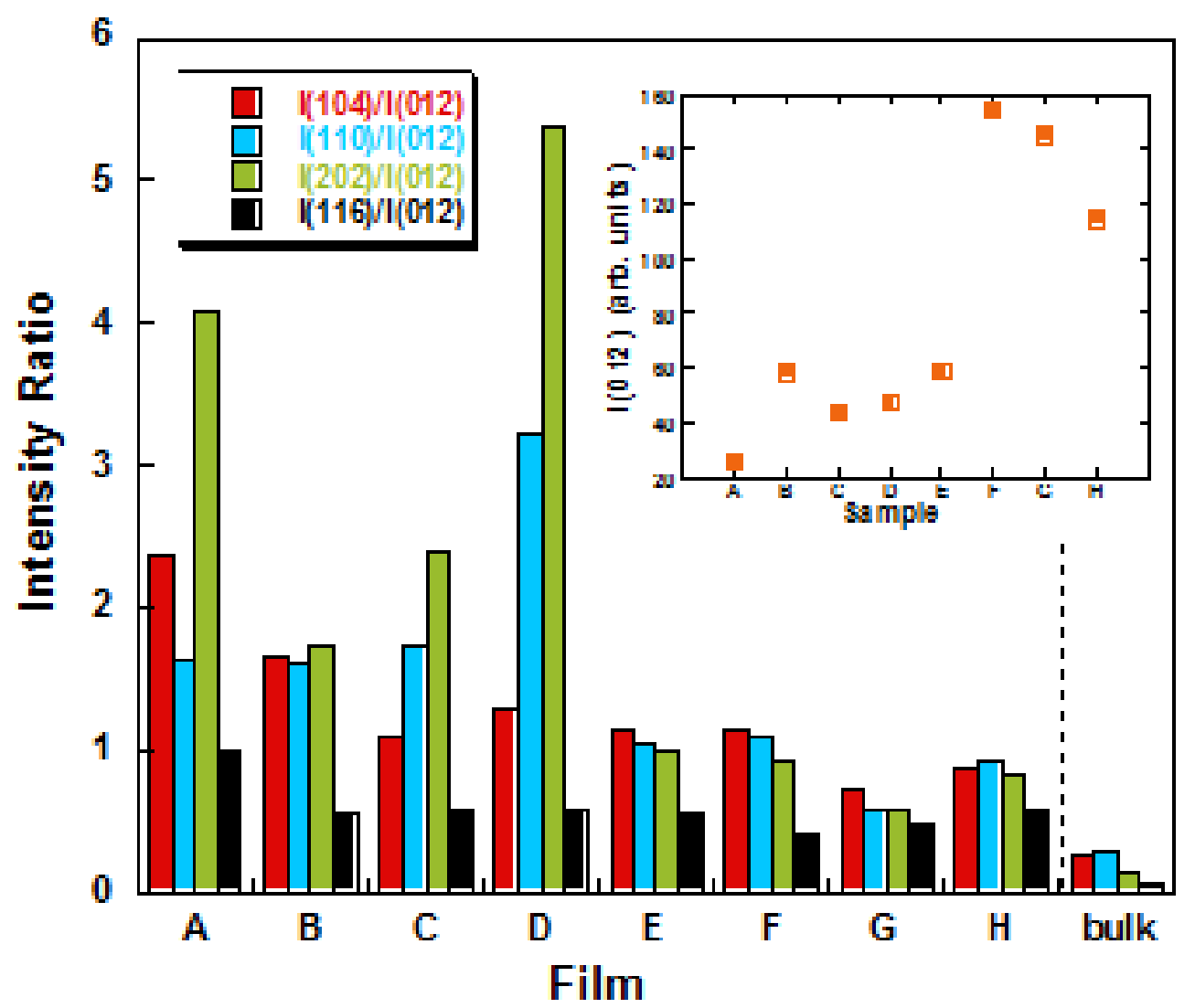

Fig. 4
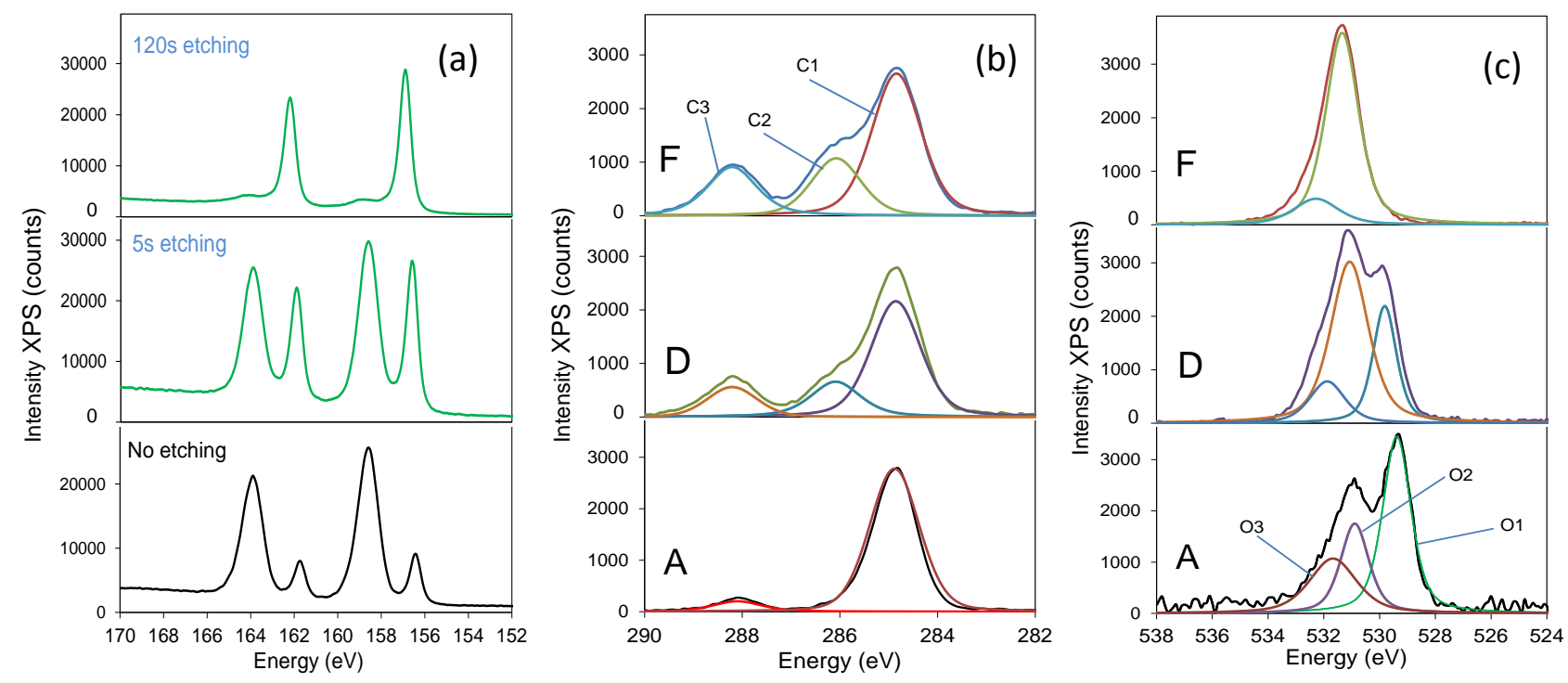

Fig. 5 

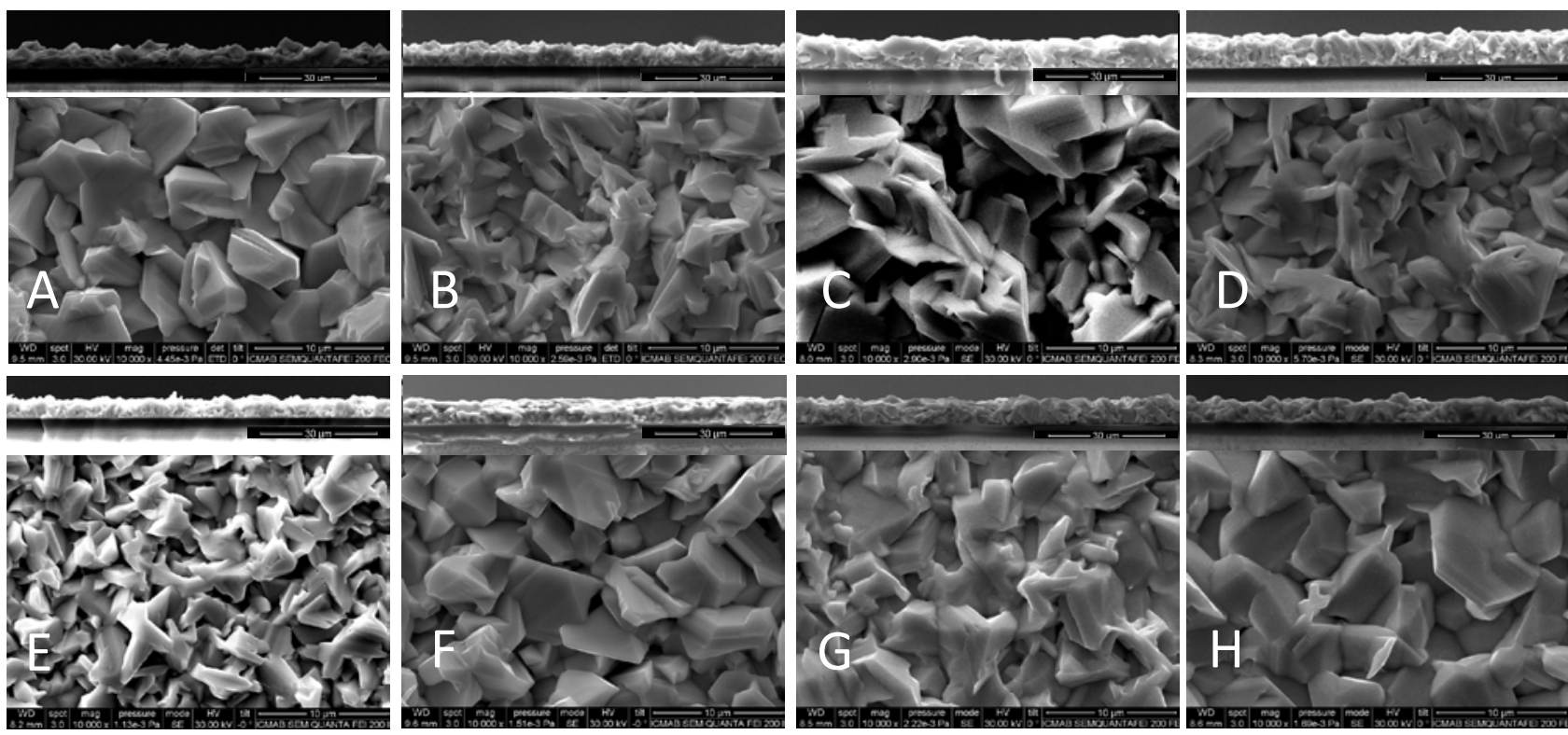

Fig. 6

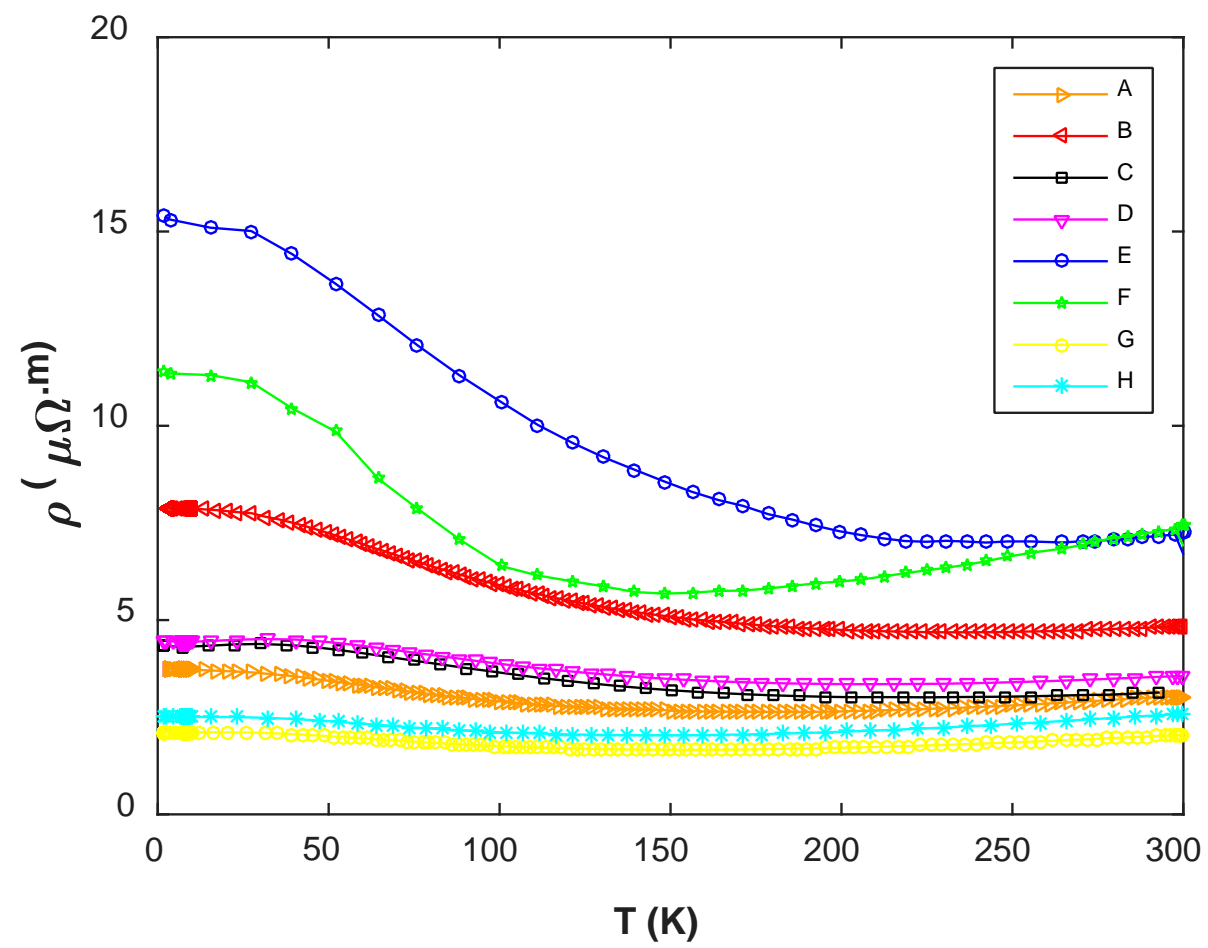

Fig. 7 

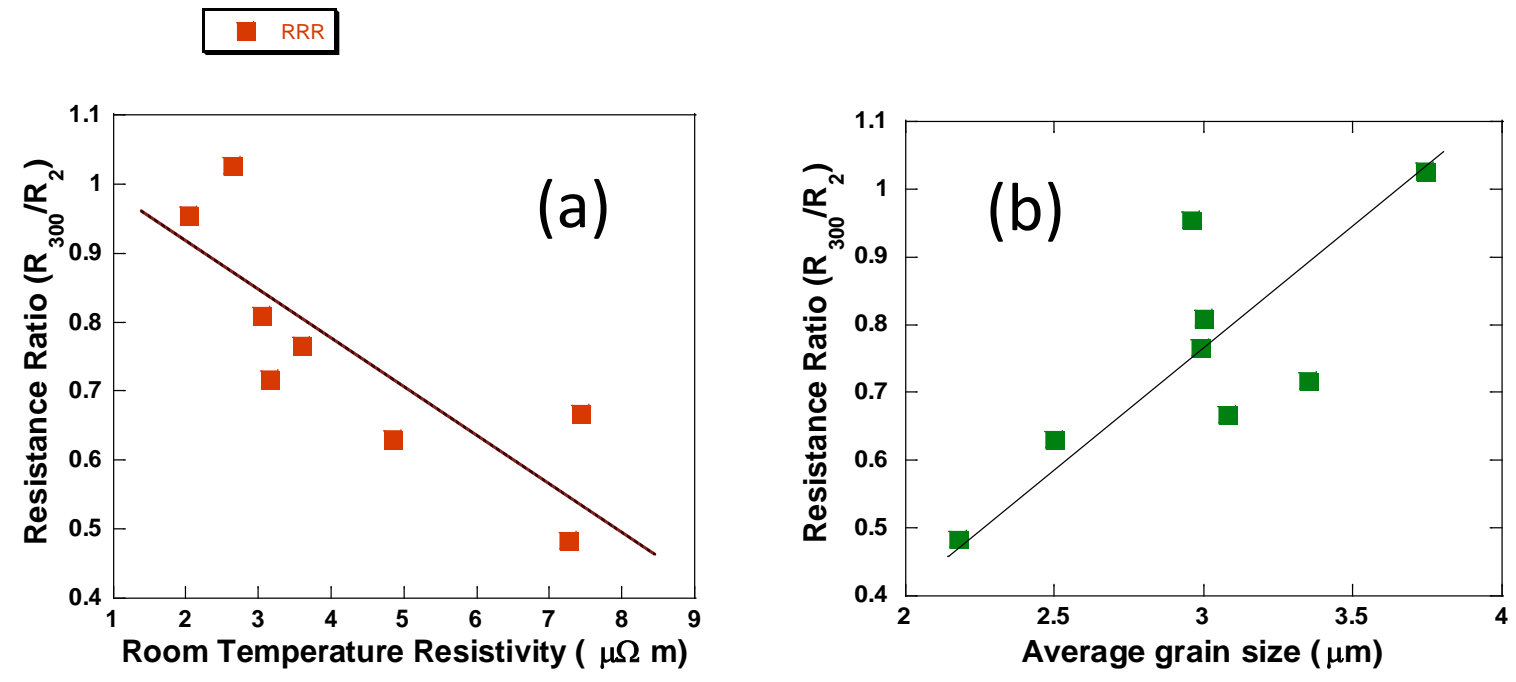

Fig. 8

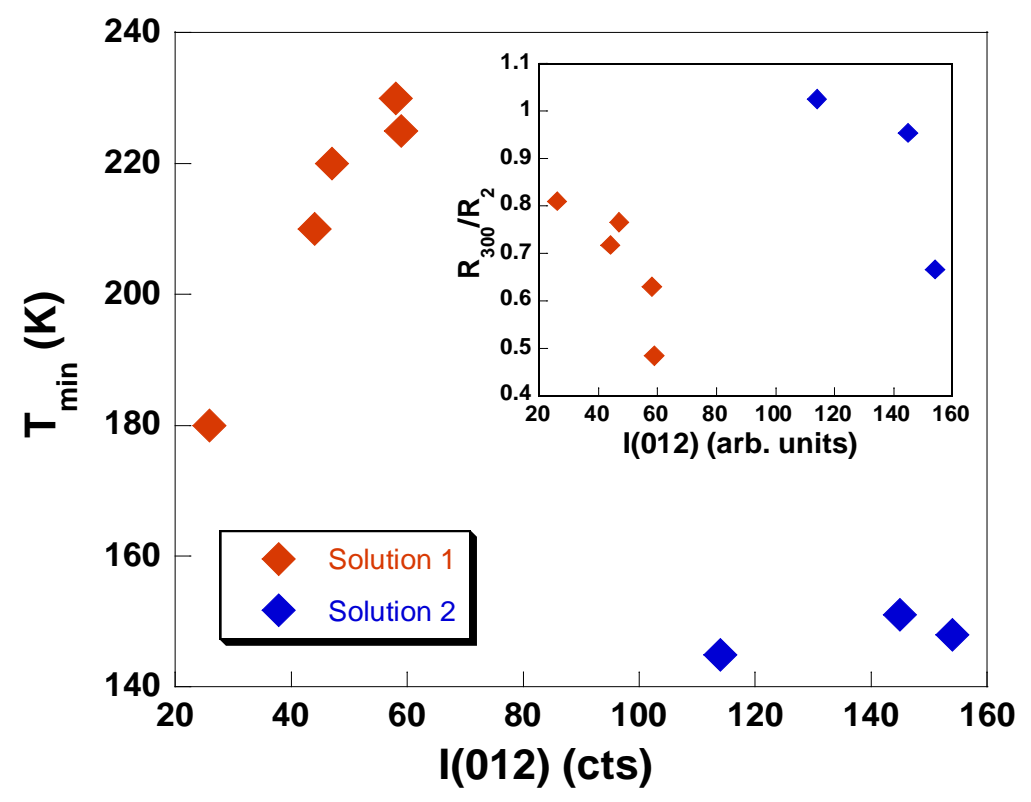

Fig. 9 